\title{
Musical rhythms in heart period dynamics: a cross-cultural and interdisciplinary approach to cardiac rhythms
}

\author{
H. BETTERMANN, ${ }^{1}$ D. AMPONSAH, ${ }^{2}$ D. CYSARZ, ${ }^{1}$ AND P. VAN LEEUWEN ${ }^{3}$ \\ ${ }^{1}$ Department of Clinical Research, Gemeinschaftskrankenhaus, 58313 Herdecke, Germany; \\ 2Biochemistry Department, University of Sciences and Technology, Kumasi, Ghana; \\ and ${ }^{3}$ Research and Development Center for Microtherapy, 44799 Bochum, Germany
}

\begin{abstract}
Bettermann, H., D. Amponsah, D. Cysarz, and P. Van Leeuwen. Musical rhythms in heart period dynamics: a cross-cultural and interdisciplinary approach to cardiac rhythms. Am. J. Physiol. 277 (Heart Circ. Physiol. 46): H1762-H1770, 1999.-The purpose of this study was to expand classic heart period analysis methods by techniques from ethnomusicology that explicitly take complex musical rhythm principles into consideration. The methods used are based on the theory of African music, the theory of symbolic dynamics, and combinatorial theory. Heart period tachograms from 192 24-h electrocardiograms of 96 healthy subjects were transformed into binary symbol sequences that were interpretable as elementary rhythmic (percussive) patterns, the time lines in African music. Using a hierarchical rhythm pattern scheme closely related to the Derler Rhythm Classification (from jazz theory), we calculated the predominance and stability of pattern classes. The results show that during sleep certain classes, specific to individuals, occurred in a cyclically recurrent manner and many times more often than expected. Simultaneously, other classes disappeared more or less completely. Moreover, the most frequent classes obviously originate from phase-locking processes in autonomic regulation (e.g., between respiratory and cardiaccycles). In conclusion, the new interdisciplinary method presented here demonstrates that heart period patterns, in particular those occurring during night sleep, can be interpreted as musical rhythms. This method may be of great potential use in music therapy research.
\end{abstract}

heart rate variability; symbolic dynamics; African music; rhythm classification; rhythm patterns; respiratory sinus arrhythmia; music therapy; cardiorespiratory synchronization

FOR MANY YEARS rhythm perception and the ability to reproduce musical rhythms have been a main topic in music psychology research (10, 16, 18, 23, 27-29, 34, 36). One question was, How are musical rhythms represented internally? E ven el ectroencephalogram (25) or positron emission tomography (40) registrations have been used to discover structures of perceived music in the mind.

On the other hand, physiologists are confronted with a huge variety of complex rhythms in physiological time series, which have often been compared with music but only to underline the high-dimensional complexity in physiological time series (4). However, as yet no investigation has revealed the link between complex

\footnotetext{
The costs of publication of this article were defrayed in part by the payment of page charges. The article must therefore be hereby marked "advertisement" in accordance with 18 U.S.C. Section 1734 solely to indicate this fact.
}

physiological and musical rhythms. Only entrainment phenomena (15) or the physiological response to auditory stimuli $(31,37)$ have been reported. The question remains unanswered: Are complex musical rhythms represented physiologically?

Apart from various definitions of "rhythm" in different disciplines, the term "musical rhythm" has a totally different significance cross-culturally with respect to its role in musical compositions. In particular, in many regions of the less industrialized world, the relationship between rhythms of life and music is much closer than that in the western world. Ethnological studies have revealed these relationships in detail (7). A better understanding of rhythm perception and rhythm cognition has been an important key to time perception (11) as well as to the complex inner dynamic of humans. In nonwestern cultures, time is often more personal than public $(1,2)$ : time perception is polychronic and closely linked with the complexity of "internal clocks." People are less determined by the need to synchronize with external clocks, which can result in time uniformity and monochronicity of time perception. This can be one reason why musical rhythms, which are the creative expressions of time, are traditionally more complex in nonwestern cultures. They are often polymetric, polyrhythmic, and asymmetric instead of monometric, monorhythmic, and symmetric like the bar-orientated European music. For further reading, the reader is referred to the rel evant literature $(20,21,24)$.

In this paper, we apply the compositional rhythm principles of African music to the analysis of cardiac time series. The fundamentals of these principles, which have also largely influenced the development of contemporary rhythm-oriented music in the Americas (e.g., Latin or jazz music), reach back several thousand years and are consequently a main issue in ethnomusicological research $(20,21)$. Unfortunately, in most of today's popular music, the huge variety of African music styles and their compositional principles have been lost.

Particularly in many African cultures, the relation between music and the human heartbeat is emphasized, and very often music is symbolically called the heartbeat of Africa. This analogy is not far-fetched, because African music is like the heartbeat, pulsating and characterized by cyclically repeated complex rhythm patterns. (A single "African music" actually does not exist. We use this term solely to delimit most common African music styles from the most common styles in European classical and contemporary music.) From the point of view of African cultures, music rises directly from the inner dynamic of human beings, 
which is symbolized and brought forth by the human heartbeat. ${ }^{1}$ African music would seem to provide a good basis for a novel approach to the analysis and better understanding of cardiac rhythms. We therefore first focus on somesimple basic principles of African musicology (21).

Theory of African music. Rhythm complexity, contrast, and cyclicity are the most important principles in African music compositions. European music theory is not suited to take these phenomena of African music into account. Thus new terms and a different notation were needed to accommodate polyrhythmic African music. Among others, some of these terms are elementary pulse, cycle (also pulse amount or cyclic pattern), pulse number (also form number), and impulse number, as well as pattern, time line pattern (also time line or set span; Ref. 3), beat, off-beat phrasing, cross rhythm, and interlocking. Only some of theseterms can be explained briefly; this explanation is provided without the claim of being exhaustive. Figure 1 illustrates the definitions used.

The elementary pulse is the basic and smallest, i.e., nondivisible time unit of musical rhythm consisting of actions and nonactions/beats and rests. Rhythm cycles are multiples of the elementary pulses, usually 8,12 , 16, or 24 pulses (cf. 12-pulse example in Fig. 1). The number of pulses in one cycle is termed the pulse number. All beats, accents, or, more generally, impulses in one cycle fall on the elementary pulses, and their sum total in one cycle is called the impulse number. Cycles can consist of one or more patterns, the latter being indivisible autonomic elements that can be found on several levels or timescales in music compositions. In general, patterns are perceived as a whole and are the rhythm units in African music. In this paper, we adapt these terms to demonstrate the possible similarity of African musical and physiological temporal structures.

Symbolic dynamics. With its concept of symbolic dynamics (14), nonlinear dynamics theory provides a tool for musical rhythm analysis of cardiac time series. For this kind of application the heart period tachogram must betransformed into symbol sequences that can be interpreted as either musical notes or rhythmic (percussive) patterns. We decided to apply the latter.

The transformation of heart rate or heart period series into symbolic sequences is not new. Kurths et al. $(22,39)$ used this instrument with various transforma-

\footnotetext{
1 "What is rhythm? It is the architecture of the being, the internal dynamism which gives him form, the system of waves which he emits in relation to Others, the pure expression of vital force. Rhythm is the vibratory shock, the force which, through the senses, seizes us at the root of our being. It is expressed through the most material and most sensual means: lines, surfaces, colours and volumes in architecture, sculpture and painting; accents in poetry and music; but in doing this it guides all that is concrete towards the light of the mind. With the African Negro, rhythm enlightens the spirit to the precise extent to which it is embodied in sensuality. African dancing abhors physical contact. But watch the dancers. If their lower limbs are shaken with the most sensual tremors, their heads are sharing in the serene beauty of the masks, of the Dead" (35).
}

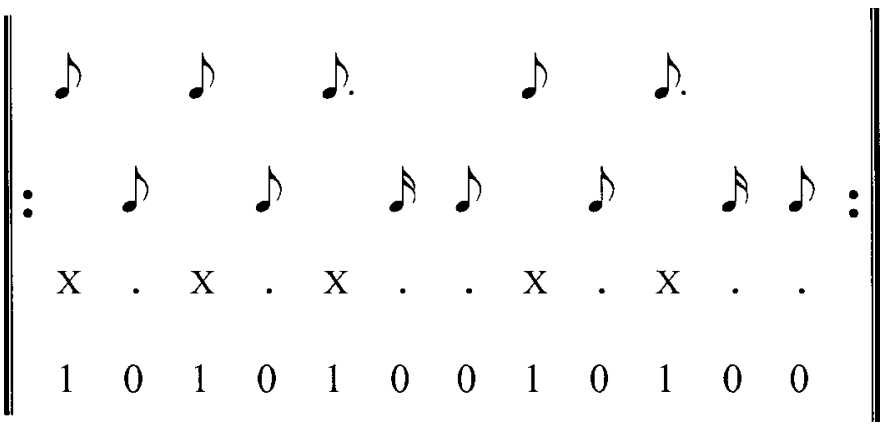

Fig. 1. Three alternative musical notations of the inyimbo rhythm (1st, 3rd, and 4th rows), which is performed by cyclic repetition of this asymmetric pattern, consisting of 12 elementary pulses (= pulse number) including 5 beats (= impulse number) plus 7 rests. In this example, the pulses correspond to 16th notes. The complementary 12-pulse pattern with 7 impulses in 2nd row is called omele and is also a popular timeline pattern in African music.

tion techniques to facilitate heart period analysis. Each of their symbolization techniques was implemented for a particular approach. The main difference from our approach is that they constructed and analyzed the symbolic sequences solely from the point of view of complexity analysis (e.g., using renormalized entropy). Musical rhythmic aspects were not taken into consideration.

Rhythm patterns with pul se numbers from 3 to 8: the Derler Rhythm Classification. In our approach, the pattern analysis is restricted to a maximum pulse number of eight heartbeats. Longer cycles were ignored because they are largely affected by external modulations and their cyclic character is generally significantly disturbed and difficult to recognize.

Table 1 summarizes all binary patterns with pulse numbers from 3 to 8 . For computational use and to identify patterns that bel ong to the same pattern class, it is more practical to use their decimal (code) rather than binary representation. The rhythm scheme is closely related to the Derler Rhythm Classification, which was developed further by Thomas Pfob and Alfons Dauer and published by Alfons Dauer in 1988 (9). Because we devel oped the rhythm scheme independently, a slightly different notation is used.

The total number of all possible combinations of the symbols 0 and 1 (musically interpreted, e.g., as rest and beat), which is $8+16+32+64+128+256=504$, can be reduced to 42 musically relevant pattern classes (= equivalent classes; Ref. 9).

Thefirst step was to exclude patterns containing only beats or rests ( 12 altogether). The remaining patterns were combined according to the following two redundancy principles. 1) All patterns that are identical after rotation (= transposition; Ref. 9) were merged to pattern classes. The $\mu$ (class) is the number of patterns bel onging to one pattern class (= rhythm amount; Ref. 9), e.g., $\operatorname{class}(011)=\operatorname{class}(110)=\operatorname{class}(101) ; \mu(011)=3$. In Table 1, all pattern classes are listed in the order of the decimal code of their smallest binary representative (= basic pattern; Ref. 9). 2) Additionally, complementary pattern classes resulting from the permutation of 1 and 0 were merged to one class. In Table 1 , 
Table 1. Rhythm scheme

\begin{tabular}{|c|c|c|c|c|c|c|c|c|}
\hline Class & Code & $\mu$ & Pattern & Class & Code & $\mu$ & Pattern & $f_{G}$ \\
\hline 1 & 1 & 3 & 001 & -1 & 3 & 3 & 011 & 1.22 \\
\hline 2 & 1 & 4 & 0001 & -2 & 7 & 4 & 0111 & 0.88 \\
\hline 3 & 3 & 4 & 0011 & -3 & (3) & 0 & 0011 & 1.12 \\
\hline 4 & 5 & 2 & 0101 & -4 & (5) & 0 & 0101 & 2.12 \\
\hline 5 & 1 & 5 & 00001 & -5 & 15 & 5 & 01111 & 0.52 \\
\hline 6 & 3 & 5 & 00011 & -6 & 7 & 5 & 00111 & 0.81 \\
\hline 7 & 5 & 5 & 00101 & -7 & 11 & 5 & 01011 & 1.86 \\
\hline 8 & 1 & 6 & 000001 & -8 & 31 & 6 & 011111 & 0.26 \\
\hline 9 & 3 & 6 & 000011 & -9 & 15 & 6 & 001111 & 0.49 \\
\hline 10 & 5 & 6 & 000101 & -10 & 23 & 6 & 010111 & 1.28 \\
\hline 11 & 7 & 6 & 000111 & -11 & (7) & 0 & 000111 & 0.56 \\
\hline 12 & 9 & 3 & 001001 & -12 & 27 & 3 & 011011 & 1.55 \\
\hline 13 & 11 & 6 & 001011 & -13 & 13 & 6 & 001101 & 1.68 \\
\hline 14 & 21 & 2 & 010101 & -14 & (21) & 0 & 010101 & 3.41 \\
\hline 15 & 1 & 7 & 0000001 & -15 & 63 & 7 & 0111111 & 0.11 \\
\hline 16 & 3 & 7 & 0000011 & -16 & 31 & 7 & 0011111 & 0.26 \\
\hline 17 & 5 & 7 & 0000101 & -17 & 47 & 7 & 0101111 & 0.74 \\
\hline 18 & 7 & 7 & 0000111 & -18 & 15 & 7 & 0001111 & 0.32 \\
\hline 19 & 9 & 7 & 0001001 & -19 & 55 & 7 & 0110111 & 1.01 \\
\hline 20 & 11 & 7 & 0001011 & -20 & 29 & 7 & 0011101 & 1.15 \\
\hline 21 & 13 & 7 & 0001101 & -21 & 23 & 7 & 0010111 & 1.14 \\
\hline 22 & 19 & 7 & 0010011 & -22 & 27 & 7 & 0011011 & 1.48 \\
\hline 23 & 21 & 7 & 0010101 & -23 & 43 & 7 & 0101011 & 2.94 \\
\hline 24 & 1 & 8 & 00000001 & -24 & 127 & 8 & 01111111 & 0.04 \\
\hline 25 & 3 & 8 & 00000011 & -25 & 63 & 8 & 00111111 & 0.13 \\
\hline 26 & 5 & 8 & 00000101 & -26 & 95 & 8 & 01011111 & 0.36 \\
\hline 27 & 7 & 8 & 00000111 & -27 & 31 & 8 & 00011111 & 0.17 \\
\hline 28 & 9 & 8 & 00001001 & -28 & 111 & 8 & 01101111 & 0.54 \\
\hline 29 & 11 & 8 & 00001011 & -29 & 61 & 8 & 00111101 & 0.62 \\
\hline 30 & 13 & 8 & 00001101 & -30 & 47 & 8 & 00101111 & 0.65 \\
\hline 31 & 15 & 8 & 00001111 & -31 & (15) & 0 & 00001111 & 0.16 \\
\hline 32 & 17 & 4 & 00010001 & -32 & 119 & 4 & 01110111 & 0.64 \\
\hline 33 & 19 & 8 & 00010011 & -33 & 59 & 8 & 00111011 & 1.02 \\
\hline 34 & 21 & 8 & 00010101 & -34 & 87 & 8 & 01010111 & 1.94 \\
\hline 35 & 23 & 8 & 00010111 & -35 & 29 & 8 & 00011101 & 0.76 \\
\hline 36 & 25 & 8 & 00011001 & -36 & 55 & 8 & 00110111 & 0.96 \\
\hline 37 & 27 & 8 & 00011011 & -37 & 39 & 8 & 00100111 & 1.03 \\
\hline 38 & 37 & 8 & 00100101 & -38 & 91 & 8 & 01011011 & 2.47 \\
\hline 39 & 43 & 8 & 00101011 & -39 & 53 & 8 & 00110101 & 2.58 \\
\hline 40 & 45 & 8 & 00101101 & -40 & (45) & 0 & 00101101 & 2.53 \\
\hline 41 & 51 & 4 & 00110011 & -41 & (51) & 0 & 00110011 & 1.46 \\
\hline 42 & 85 & 2 & 01010101 & -42 & (85) & 0 & 01010101 & 5.60 \\
\hline
\end{tabular}

$\mu$, No. of patterns in class; $f_{G}$, normalized relative frequency for an artificially generated Gaussian-distributed random R-R tachogram. See text for details.

complementary classes (also with their smallest binary representative) are in the same line and have the same class number except for sign.

Combinatorial theory. The rhythm classification can be understood as a typical application of combinatorial analysis that provides a profound mathematical background for the above elaboration. According to this theory, the first redundancy principle corresponds to the definition of a cyclic group $\mathrm{C}_{m}$ of "shifts in origin," which act on a set $P_{m}$ of sequences with period $m$. The shifts can be compared with the rotation of a necklace which, in our case, is made from $\mathrm{m}$ beads of two different kinds $(12,13)$. The second principle can be interpreted as the definition of a symmetric group $\mathrm{S}_{2}$ of permutations that here simply means exchanging 1 and 0 . The combination of the two principles results in the symmetry transformation group $\mathrm{C}_{\mathrm{m}} \times \mathrm{S}_{2}$, which establishes a decomposition of $\mathrm{P}_{\mathrm{m}}$ into equivalence classes or symmetry types of periodic sequences. The number of elements in a specific class is given by the sum of the $\mu$ values in each row of Table 1 . Gilbert and Riordan (12) derived a formula for the number of symmetry types with respect to the period $\mathrm{m}$. According to the data in Table I of their paper and considering the fact that we ignored patterns consisting only of 1 or 0 , the number of types (equival ence classes) with a period, e.g., from $m=3-12$, are 1, 3, 3, 7, 9, 19, 29, 55, 93, and 179. In total, these are 398 pattern classes needed to cover all binary rhythm patterns with pulse numbers from 3 to 12, and 42 pattern classes for pulse numbers from 3 to 8 .

\section{METHODS}

Subjects. The baseline el ectrocardiograms (ECGs) from the Cardiodoron study (8) were used as the data resource. The study included 100 healthy subjects and was carried out at the Gemeinschaftskrankenhaus, Herdecke, Germany, from J anuary to August 1997. Because of missing data, four subjects were excluded from this retrospective analysis. For the remaining 96 subjects (age 20-41 yr, mean \pm SD $28 \pm 7$ yr; 45 men, 51 women), two successive 24-h ECGs (A and B) were available. The sleep times could be determined from the subjects' diaries.

Construction of symbol ic sequences. Oxford FD3 solid-state recorders with simultaneous $\mathrm{R}$ wave detection were used for the 24-h ECG registration. Visual inspection of the automatically detected $R$ waves was performed on an Oxford Excel ECG-Analyzer. The R times of all beats were written to a binary data file that was then exported to a Pentium PC for further analysis.

The times between successive $R$ waves ( $R-R$ intervals) were calculated in milliseconds, and they form the R-R tachogram. Herein artifacts and ectopic beats were marked with the value 9999. Next, the differences of all successive R-R intervals were calculated. These differences formed the differential R-R tachogram, which was directly transformed into a binary symbolic sequence as follows (see also Fig. 2). 1) R-R differences smaller than zero are marked with 1 , which corresponds to an acceleration of the heartbeat. 2) R-R differences greater than or equal to zero are marked with 0 , which corresponds to a deceleration of the heartbeat. 3) Missing values or R-R differences that are corrupted by artifacts or ectopic beats are marked with 9 .

Pattern frequency and stability. The computational routine classtat generally calculates the relative frequency of all 42 pattern classes for any time interval during the day. In the present application, classtat was applied to all successive 1-h intervals of the entire 24-h period.

A window with a variablelength of $m=3 \ldots .8$ symbols (pulse number) is moved in equidistant steps of one heartbeat over

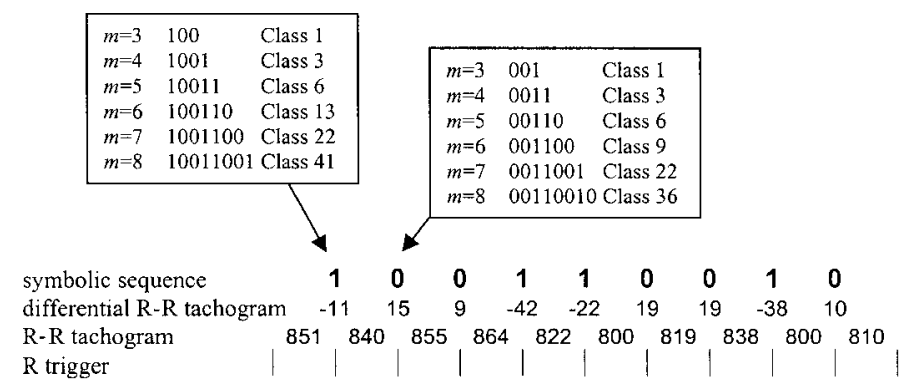

Fig. 2. Example of construction of a symbolic sequence and determination of corresponding pattern classes. $\mathrm{m}$, Pulse number. 
the 1-h period, providing five patterns with different pulse numbers for each heartbeat (see Fig. 2). E very pattern, which can be assigned to a pattern class from 1 to 42 , is counted by incrementing the corresponding component of a 42-dimensional vector. To make the frequency values of all pattern classes comparable to each other, for each hour, the absolute frequencies $F$ (dlass) are divided by the rhythm amount $\mu$ (dass) as well as by the total number of all registered patterns $\mathrm{N}(\mathrm{m})$ with the same pulse number $\mathrm{m}$ and are multiplied by $2^{m}$, the number of all possible m-dimensional binary combinations

$$
f(\text { class })=\frac{F(\text { class }) \cdot 2^{m}}{N(m) \cdot \mu(\text { class })}
$$

The resulting normalized relative frequency $f$ (class) is $<1$ if class is less frequent and $>1$ if class is more frequent than would be expected in equally distributed random symbol sequences.

The classtat routine al so cal culates the mean step number s(class) for which the class of the pattern remains unchanged, when the window is moved stepwise over the entire symbol sequence; s(class) is termed cyclic pattern stability.

Pattern predominance. To quantify the predominance of patterns, the difference of the mean of the three greatest $f$ values and the mean of the three smallest $f$ values is calculated for each 1-h time interval, giving 24 values/day. This measure is termed pattern predominance (PP), and it serves as an information reduction of the entire 24-h ECG. Here the pattern stability plays no role.

Coupling patterns. Some typical patterns are probably the result of coupling processes (see Table 2 ). The patterns can occur if the phases of the heart period and a heart period modulating harmonic oscillation are locked. Figure 3 demonstrates this by way of example for a 7:2 phase locking. These patterns will likely correspond to respiratory sinus arrhythmia (RSA), the strongest heart period modulation, which primarily occurs because of interaction in the central nervous system of the cardiac and respiratory systems. On average, the number of heart periods per respiratory cycle is approximately four in untrained healthy adults at rest. This can be easily shown if one counts all heartbeats and respiratory cycles during night sleep and calculates the quotient. Consequently, phase locking ratios near 4:1 (e.g., 7:2, 9:2, 3:1, 5:1) between cardiac and respiratory cycle should be predominant at rest if phase locking occurs. To test this hypothesis, we paid particular attention to the pattern classes listed in Table 3. Here the following pattern classes were further merged: classes 1 and 12; classes 2 and 42; classes 3 and 41; classes 4, 14, and 42 (see al so Table 1).

Musical rhythm patterns. Every possible pattern can also be seen as a musical rhythm pattern. However, some musical

Table 2. Patterns that can originatefrom $x$ :y phase locking between cardiac cycl ex and some modulating cycley (e.g., respiratory cycle)

\begin{tabular}{llll}
\hline \hline $3: 1 \rightarrow 101$ & $4: 1 \rightarrow 1001$ & $5: 1 \rightarrow 11001$ & $6: 1 \rightarrow 110001$ \\
$3: 2 \rightarrow 011$ & $4: 2 \rightarrow 0101$ & $5: 2 \rightarrow 10101$ & $6: 2 \rightarrow 101101$ \\
$3: 3 \rightarrow 111$ & $4: 3 \rightarrow 0011$ & $5: 3 \rightarrow 01011$ & $6: 3 \rightarrow 010101$ \\
$3: 4 \rightarrow 101$ & $4: 4 \rightarrow 1111$ & $5: 4 \rightarrow 00111$ & $6: 4 \rightarrow 011011$ \\
$3: 5 \rightarrow 011$ & $4: 5 \rightarrow 1001$ & $5: 5 \rightarrow 11111$ & $6: 5 \rightarrow 000111$ \\
$7: 1 \rightarrow 1110001$ & $8: 1 \rightarrow 11100001$ & $9: 1 \rightarrow 111100001$ & $10: 1 \rightarrow 1111000001$ \\
$7: 2 \rightarrow 1001101$ & $8: 2 \rightarrow 10011001$ & $9: 2 \rightarrow 110011001$ & $10: 2 \rightarrow 1100111001$ \\
$7: 3 \rightarrow 1010101$ & $8: 3 \rightarrow 10100101$ & $9: 3 \rightarrow 101101101$ & $10: 3 \rightarrow 1001001101$ \\
$7: 4 \rightarrow 0101011$ & $8: 4 \rightarrow 01010101$ & $9: 4 \rightarrow 101010101$ & $10: 4 \rightarrow 1010110101$ \\
$7: 5 \rightarrow 0110011$ & $8: 5 \rightarrow 01001011$ & $9: 5 \rightarrow 010101011$ & $10: 5 \rightarrow 0101010101$ \\
\hline
\end{tabular}

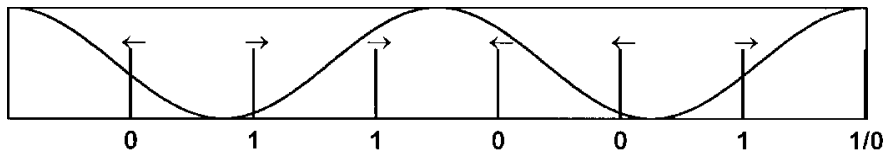

Fig. 3. Illustration of a 7:2 phase locking of heartbeat (vertical bars mark R time) and a heart period modulating harmonic oscillation (e.g., respiration); shown are the effect on the heartbeat period and its symbolic dynamic. The 2 possible binary patterns are complementary and belong to the same pattern class 22 .

patterns are more common than others. In general, distinct asymmetric time line patterns play an important role in African music. These patterns have an even pulse number $\mathrm{m}$ with $\mathrm{m} / 2-\mathrm{k}$ or with $\mathrm{m} / 2+\mathrm{k}$ beats $(\mathrm{k}=1,3 \ldots \mathrm{m} / 2-1)$. The beats and rests are distributed as equally as possible over the $\mathrm{m}$ pulses. The two asymmetric patterns for each $k$ are complementary. Dauer and Pfob (see Ref. 9) described the mathematical construction rule in detail and named the thus-constructed patterns final rhythms, because they are at the end of the Derler Rhythm Hierarchy. In our rhythm scheme, the patterns that belong to the eight-pulse pattern classes 38 and -38 are the only musically relevant asymmetric patterns. The most common time line patterns in African music are the 12-pulse pattern with five beats (101010010100) or its complementary pattern with seven beats (101011010110) (cf. Fig. 1), which are not in our scheme because of their length. Beyond that, two further pattern classes are of interest. Class 10 can be interpreted as a $2 \times 3$ polyrhythm (also called 2 against 3 cross rhythm), which is the resultant of a double meter (class 14) and a triple meter rhythm (class 12). Classes 2 and 32 can be interpreted as the well-known $4 / 4$ meter.

Surrogate data. The question of whether properties that were found in time series result from nonlinear deterministic processes can be answered by testing for nonlinearity with the method of surrogate data. In this paper, two different null hypotheses are tested with two different sets of surrogate data. The first, less restrictive null hypothesis is that the predominance of certain musical rhythm patterns is only caused by properties of the R-R distribution. This is tested by shuffling the R-R tachogram of each 10-min interval such that the distribution of the R-R values is kept but their order becomes random. The second, more restrictive null hypothesis tests more specifically for nonlinearity, using the improved surrogate data method of Schreiber and Schmitz described in detail elsewhere (33). The improved R-R surrogates have not only the same distribution but also the same power spectrum as the given R-R tachograms. Thus their

Table 3. Pattern classes and their physiological or musical interpretation

\begin{tabular}{lcc}
\hline \hline Class & $\begin{array}{c}\text { Physiological } \\
\text { Phase Locking }\end{array}$ & $\begin{array}{c}\text { Musical } \\
\text { Rhythms }\end{array}$ \\
\hline $4,14,42$ & $2: 1$ & \\
1,12 & $3: 1$ & $3 / 4$ Meter \\
3,41 & $4: 1$ & \\
6 & $5: 1$ & \\
7 & $5: 2$ & \\
11 & $6: 1$ & \\
22 & $7: 2$ & \\
23 & $7: 3$ & 4/4 Meter \\
40 & $8: 3$ & AS8 \\
2,32 & & $2 \times 3$ Polyrhythm \\
38 & &
\end{tabular}

AS8, asymmetric 8-pulse pattern. 
linear stochastic properties are approximately identical with those of the respective original data, but the nonlinearities are destroyed. Both sets of R-R surrogates were transformed into binary symbolic sequences and analyzed with the above described method.

\section{RESULTS}

Examples. Figures 4, 5, and 6 show three typical examples of the daily course of the pattern frequency $\mathrm{f}$ (class) for all 42 pattern classes (top) and of the pattern predominance PP (bottom).

Subject 23 reported sleeping from 15:15 to 16:00 and from 21:30 to 5:32. In Fig. 4, both periods are well marked by the changes of contrast of f(class). During the daytime, all patterns are more or less equally frequent, whereas at rest and during night sleep, specific patterns are markedly overrepresented. The pattern predominance increases to PP $=8.2$. The greatest contrast is revealed by pattern class 41 (00110011). It varies between $f=0.28$ (from 19:00 to 20:00) and $f=16.3$ (from 0:00 to 1:00). The higher $f$ values of class 41 evidently indicate a 4:1 phase locking of the respiratory and cardiac cycles during afternoon and night sleep.

The heartbeat of subject 100 (see Fig. 5) generated similar 4:1 phase-locking patterns that are highly overrepresented during night but no more frequent than all the other patterns during day. This "rhythmization" is shown clearly by the double-peaked time course of PP, with one maximum immediately after the subject fell asleep and the other one between 4:00 and 5:00 in the early morning. Simultaneously with the develop-
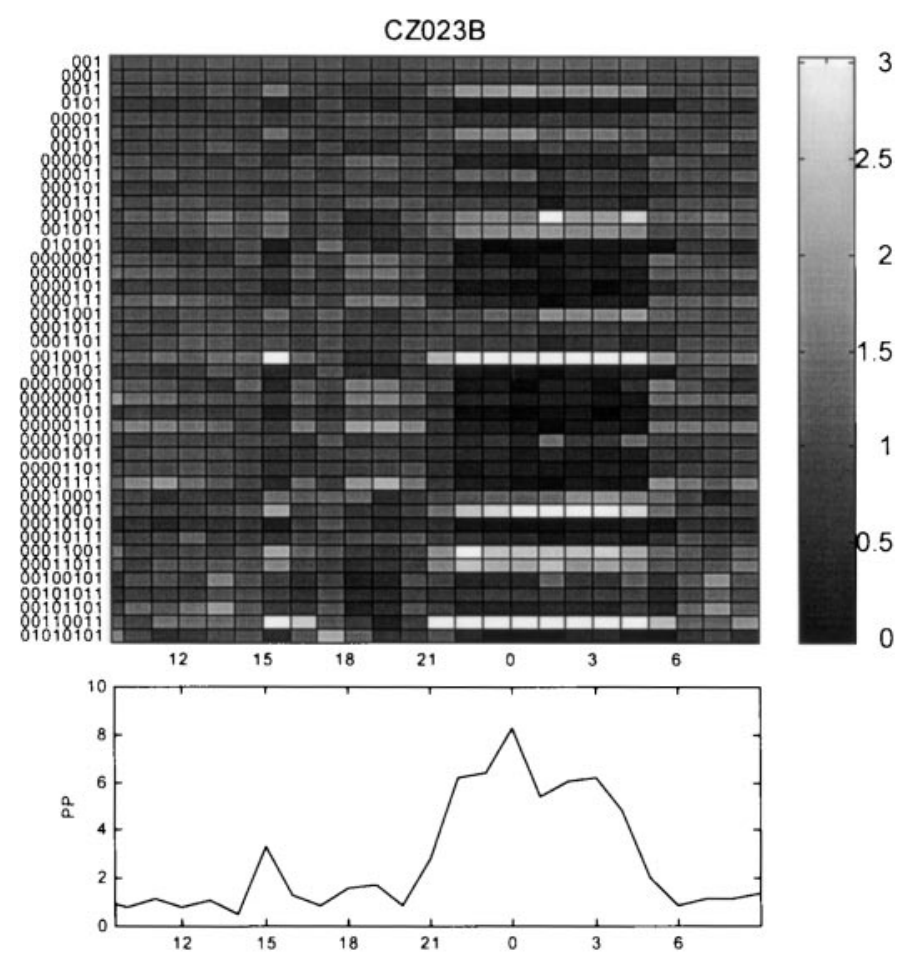

Fig. 4. Daily courses of pattern frequency $\mathrm{f}$ (class) for all 42 pattern classes (top) and of pattern predominance (PP, bottom) in electrocardiogram (ECG) of subject 23.
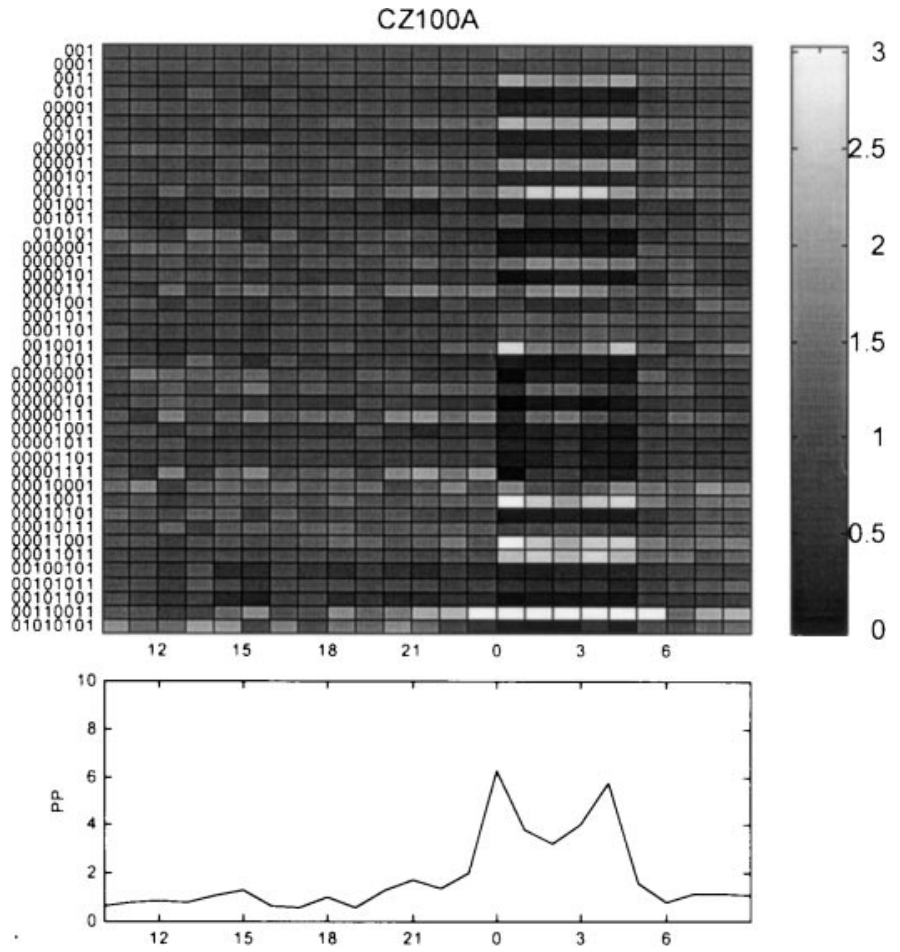

Fig. 5. Daily courses of pattern frequency f(class) for all 42 pattern classes (top) and of PP (bottom) in E CG of subject 100.

ment of specific rhythm patterns, other patterns disappear more or less completely. In the case of subject 100, disappearing patterns with $f<0.1$ are patterns of classes 24, 26, 31, 40, and 42.
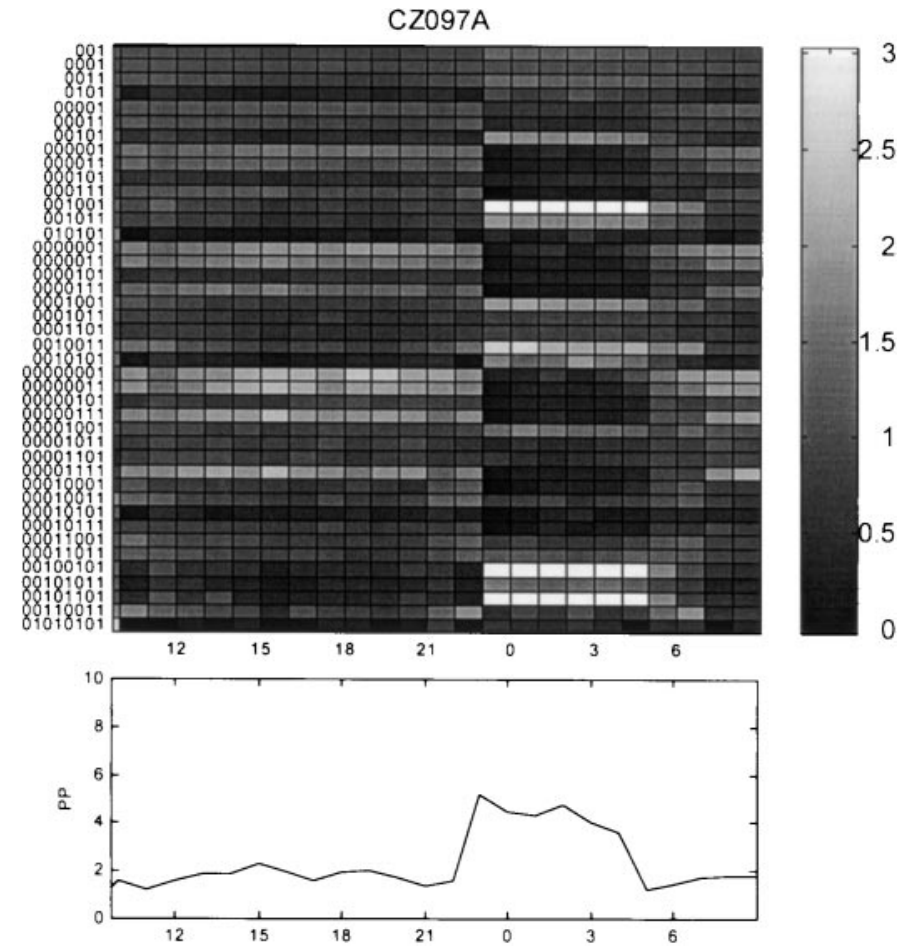

Fig. 6. Daily courses of pattern frequency $\mathrm{f}$ (class) for all 42 pattern classes (top) and of PP (bottom) in ECG of subject 97. 
The symbolic sequences of subject 97 reveal a different and unexpected rhythmization (Fig. 6). During nighttime the pattern classes 12,38 , and 40 are the most frequent, whereas class 41 is relatively infrequent. It remains to be investigated whether this phenomenon really originates from a 8:3 or 3:1 phase locking. From the music point of view, it would also be very interesting to explore the possible connection between the occurrence of class 38 and a possible preference of subjects for musical rhythms, like the asymmetric eight-pulse time line pattern, that lie behind class 38.

All 192 ECGs were analyzed in the same way and showed similar results. To determine, first, which patterns occurred preferentially during the night and, second, whether the preference of certain pattern classes was specific to an individual and reproducible, only the nighttime sleeping period between 1:00 and 5:00 was evaluated (the time was corrected according to the subject's diary if necessary).

Pattern predominance. With respect to the first question, all 1-h intervals during the nighttime with $\mathrm{f}>2$ and $s>2$ were counted. With the latter condition, the cyclic pattern stability s was explicitly taken under consideration. Table 4 shows the results. In all, 784 1-h intervals were analyzed. In five subjects no patterns were registered that fulfilled the above conditions, either in ECG A or in ECG B. According to Table 4, the heart period dynamics of the remaining 91 subjects most frequently yielded the 4:1 phase-locked pattern (35\%), followed by the 7:2 (19\%), 3:1 (17\%), 5:1 (15\%), $6: 1(12 \%)$, and 8:3 (5\%) patterns. Also, the $4 / 4$ measure pattern, which cannot be explained directly as a result of a phase-locking process, was overrepresented in 41 1-h intervals. Over all subjects, all other patterns were generated less often, but some played an important role in individuals.

Reproducibility. With respect to the question of intraindividual reproducibility, the number of 1-h intervals with an overrepresentation ( $f>2$ and $s>2$ ) of certain pattern classes was calculated separately for each individual and both recording days. In this way, we arrived at 91 pairs of numbers for each pattern class which are plotted in Fig. 7 for day A versus day B. The correlation coefficient $r$ is given, which serves as a measure for the reproducibility of the specific pattern from day A to day B within individuals. The patterns that are related to $3: 1,5: 1,6: 1$, and $8: 3$ phase locking are highly reproducible, with $r>0.8$. For the most frequently occurring phase-locking patterns (4:1 and 7:2), the values are slightly lower.

Surrogate data. Visual comparison of the pattern frequencies in original and surrogate data provided evidence that the predominance of physiological rhythm patterns was lost in shuffled data. As can be seen in Fig. 8, shuffling of R-R tachograms caused predominance of patterns of alternating 0 and 1 . This effect is numerically demonstrated in the last column of Table 1, which displays the corresponding $f$ values for an artificially generated Gaussian-distributed random R-R tachogram. Those patterns with long sequences of only 0 or 1 are per se less frequent than those with alternating 0 and 1 . F or example, class 42 with its strong 01 rhythmicity $(f=5.6)$ is the most frequent, whereas the pattern consisting of seven 0s or 1s appears most infrequently $(f=0.4)$. The value for PP of the random data is 3.94. These findings are the result of a numerical simulation. We have not yet found a mathematical theory that enables an analytic calculation of $f$ values when the distribution of values in random time series is given. However, with this numerical experiment it could sufficiently be demonstrated why and to what extent the symbolic patterns of shuffled R-R tachograms are not equally frequent.

Examining the results for the improved surrogate data, we found that the pattern frequencies could not be distinguished from those of the original data. This suggests that the pattern frequencies result primarily from thelinear properties of the R-R tachogram.

\section{DISCUSSION}

In this paper we introduce a cross-cultural and interdisciplinary approach to cardiac rhythms. It is based both on some principles of symbolic dynamics and on theoretical aspects of African music under explicit consideration of the cyclicity of rhythm patterns. The rhythm classification, based on combinatorial theory, is not new. It has been previously applied successfully in musicology to disclose important principles, in particular, of African music compositions. Here we have used the same approach in heart period analysis.

It is widely accepted as common knowledge that musical rhythms are cl osely related to the human sense of the present (11). The time span of the present is framed by the two elementary and most dominant rhythms of life, respiration and heartbeat (17). Indeed, in many cultures of the world it is a matter of course that musical rhythms are an expression of heartbeat and respiration. The aim of this study was to find quantitative links between music and physiology that support this assumed relationship. Such links would give deeper insights not only into human physiology but also into the phenomenology of music itself. In this respect, the method presented in this study should not only be seen as an additional subtle method for the analysis of heart rate variability but also as a tool for

Table 4. Number of 1-h intervals during nighttime with $\mathrm{f}>2$ and $\mathrm{s}>2$ for original and shuffled surrogate data

\begin{tabular}{|c|c|c|c|c|c|c|c|c|c|c|c|c|c|}
\hline & $2: 1$ & 3:1 & 4:1 & $5: 1$ & $5: 2$ & $6: 1$ & $7: 2$ & $7: 3$ & $8: 3$ & $4 / 4$ & AS8 & Other & Total Analyzed \\
\hline $\begin{array}{l}\text { Originals } \\
\text { Surrogates }\end{array}$ & $\begin{array}{r}8 \\
179\end{array}$ & $\begin{array}{r}130 \\
0\end{array}$ & $\begin{array}{r}275 \\
0\end{array}$ & $\begin{array}{r}121 \\
0\end{array}$ & $\begin{array}{r}14 \\
0\end{array}$ & $\begin{array}{r}96 \\
0\end{array}$ & $\begin{array}{r}145 \\
0\end{array}$ & $\begin{array}{l}2 \\
0\end{array}$ & $\begin{array}{r}37 \\
0\end{array}$ & $\begin{array}{r}41 \\
0\end{array}$ & $\begin{array}{l}9 \\
0\end{array}$ & $\begin{array}{r}138 \\
0\end{array}$ & $\begin{array}{l}784 \\
784\end{array}$ \\
\hline
\end{tabular}

Rhythms are classified according to physiological phase-locking and musical rhythm interpretations in Table 3. 


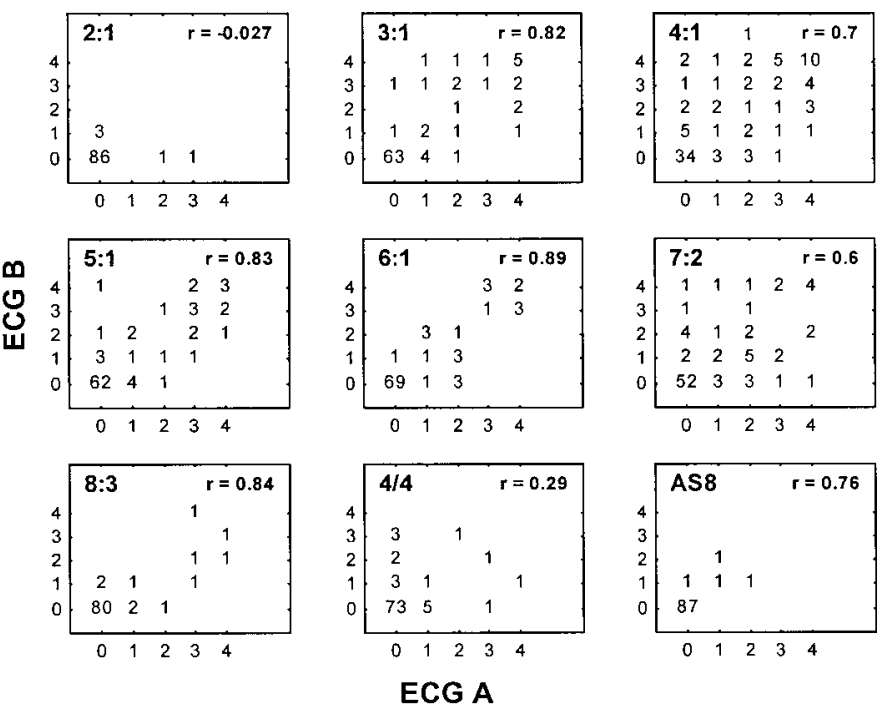

Fig. 7. Number of 1-h nighttime intervals with $f>2$ and $s>2$ for ECG A vs. ECG B. F or each pattern class thereare 91 number pairs, 1 for each subject; correlation coefficient $r$ serves as measure for reproducibility of specific pattern from day A to day B within individuals.

the systematic examination of the phenomena of heartbeat dynamics from the humanities, i.e., the musicol ogical and ethnological point of view.

The basic rhythmic element of African music is the pattern of one rhythm cycle, which serves as a timeline much like the bar in European music. This time line pattern dominates a musical composition by its strict cyclic repetition from the beginning to the end of the

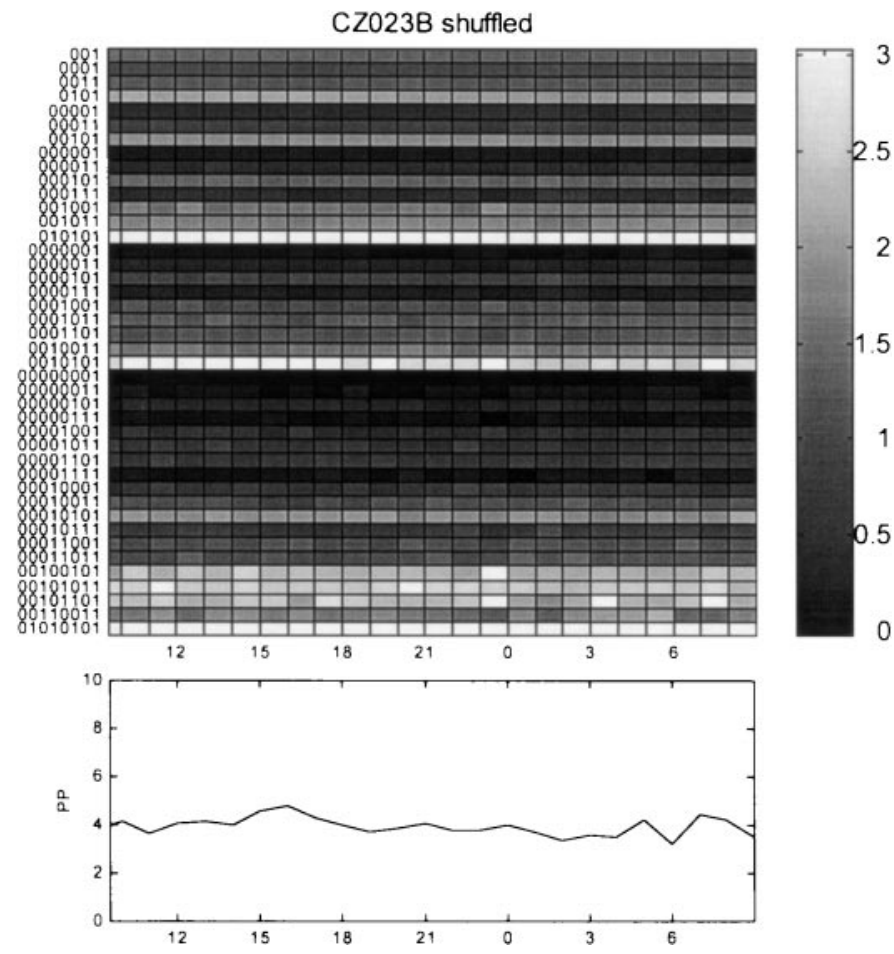

Fig. 8. Daily courses of pattern frequency f(class) for all 42 pattern classes (top) and PP (bottom) for shuffled surrogates of data for ECG of subject 23 shown in Fig. 4. piece. Beside the elementary pulsation, the time line pattern is a very important musical reference element that serves as a coordinator of African music ensembles like the baton of a philharmonic conductor. We applied the pattern concept of African music by structuring all possible time line patterns with three to eight pulses, avoiding redundancy. This resulted in a musical rhythm scheme with 42 pattern classes that is strongly related to the Derler Rhythm Classification and is based on combinatorial mathematics.

We then constructed binary symbolic patterns from the differential 24-h R-R tachogram of healthy subjects on the basis of symbolic dynamics. Together with the African music pattern concept, this allowed a musical interpretation of heart period dynamics. The al gorithm presented showed that, in some individuals during sleep, certain rhythm patterns occurred many times more often than expected. Simultaneously, other patterns disappeared more or less completely. Moreover, the musical rhythm analysis provided a tool to detect patterns that apparently originate from phase-locking processes in autonomic regulation and further allowed statements about the corresponding phase-locking ratios. For example, if RSA is present, strict phaselocking between respiratory and cardiac cycles results in cyclically repeated binary patterns of the differential symbolic heart period dynamic. In healthy subjects, 4:1 phase-locking patterns were found most frequently, followed by patterns that may result from 7:2, 3:1, and 5:1 phase locking, likely between respiratory and cardiac cycle; 9:2 phase-locking ratios were not detectable because of the implemented maximum cycle length of eight heartbeats in our algorithm. All phase-locking patterns were specific to individuals and highly reproducible.

The musical rhythmization of the heartbeat can be observed primarily during resting periods. Moreover, in most cases the predominance of musical rhythms changed at least two times abruptly during night sleep, which suggests that the quality of sleep largely influences the cardiac rhythmicity. On the other hand, we were very surprised that the previously observed increase of heart period complexity during sleep $(6,38)$ is obviously accompanied by a contradictory augmentation of rhythmic processes. Indeed, in certain 1-h data sets we observed that both the approximate entropy, as a measure for compl exity, as well as therhythm pattern predominance were notably increased. Further experiments, e.g., with model data, may help explain this phenomenon. In particular, it would be interesting to compare regularity statistics of symbolic sequences with the predominance and the cyclic recurrence of rhythm patterns. In this respect, we suggest taking up the ideas of Pincus and Singer (26), who have proposed a method to quantify randomness and degrees of irregularity, among other things, of short binary sequences of clinical data [alternatively, see also Raab and Ebner (29)].

In our data, the generation of surrogate R-R tachograms showed, first, that the detected pattern predominance is an intrinsic physiological property and, sec- 
ond, that this property is probably caused by autocorrelations and not by deterministic nonlinearities. As a result, physiological pattern predominance must be inherent in the power spectra of R-R time series. However, as the spectra do not reveal this "musicality" of the heartbeat, musical rhythms remain hidden when only the spectral characteristics of heart rate variability are analyzed.

In the past, cardiac respiratory coupling has been a main topic of many technically and/or mathematically elaborated investigations $(19,30,32)$, which were all based on the simultaneous registration of heart period and respiration to exhibit the couplings of the cardiac respiratory control loop. In this study it could be shown that the unsophisticated "musical" rhythm analysis of the differential symbolic R-R series may represent a more easily applicable tool to detect and to analyze phase-locking ratios between cardiac and respiratory cycles. Because this tool provides no certainty that the detected cyclic rhythm patterns really originate from a phase locking of cardiac and respiratory cycles, further studies are needed to confirm this presumed relationship.

In some sense, the musical heart rhythm analysis is a simplification of the electrocardiographic respiratory rate detection method we introduced in an earlier publication (5): after the R-R tachogram was band-pass filtered in the respiratory band, the proposed method was based also on the evaluation of the differential heart period dynamic. However, it disregarded the power of the dynamic's cyclic behavior and the steady recurrence of identical symbolic rhythm patterns that, from the music point of view, are the most important characteristics of rhythms in music and life.

In the future, the search for fundamental links between musical and physiological rhythms can give rise to truly interdisciplinary research. This may provide wider aspects for rhythm and time perception and can also yiel $d$ a better understanding of time structures in human life. A possible strategy could be, first, to make physiological rhythms audible via suitable symbolic data transformations into audio computer files and second, to analyze the psychophysiological response to the perception of such personal physiological rhythms. These experiments should include percussion (at least finger tapping) or dance to reproduce the rhythms and should pursue the question whether distinct physiological rhythms can be specifically amplified. This positive physioacoustic feedback or rhythmic resonance may be of great potential use, in particular, for the evaluation of music therapies.

This work was financially supported by WELEDA, SchwäbischGmünd.

Address for reprint requests and other correspondence: $\mathrm{H}$. Bettermann, Dept. of Clinical Research, Gemeinschaftskrankenhaus, 58313 Herdecke, Germany (E-mail: h.better@t-online.de).

Received 9 November 1998; accepted in final form 10 J une 1999.

\section{REFERENCES}

1. Aldridge, D. A phenomenological comparison of the organization of music and the self. Arts Psychother. 16: 91-97, 1989.
2. Aldridge, D. Physiologische Veränderungen beim Spielen improvisierter Musik-einige Vorschläge für Forschungsarbeiten. Musikther. Umsch. 3: 174- 186, 1992.

3. Anku, W. Towards a cross-cultural theory of rhythm in african drumming. In: Intercultural Music, edited by C. T. Kimberlin and A. Euba. Bayreuth, Germany: Breitinger, 1995, vol. I, p. 167202.

4. Appel, M. L., R. D. Berger, J . P. Saul, J . M. Smith, and R. J . Cohen. Beat to beat variability in cardiovascular variables: noise or music? J . Am. Coll. Cardiol. 14: 1139-1148, 1989.

5. Betterman, H., P. Engelke, P. Van Leeuwen, and C. Heckmann. Determination of respiratory rate on the basis of respiratory sinus arrhythmia. Biomed. Tech. (Berl.) 41: 319- 323, 1996.

6. Betterman, H. P., and P. Van Leeuwen. Evidence of phase transitions in heart period dynamics. Biol. Cybern. 78: 63-70, 1998.

7. Chernoff, J . M. African Rhythm and African Sensibility. Chicago: Univ. of Chicago Press, 1979.

8. Cysarz, D., T. Schürholz, H. Bettermann, and H. C. Kümmel. Heart rate variability as a subtle method for observing complex reactions of rhythmical processes. Arzneimittelforschung. In press.

9. Dauer, A. Derler 1: ein System zur Klassifikation von Rhythmen. Musiktheoretische und musikhistorische Aspekte. J azzforschung 20: 117-154, 1988.

10. Drake, C. Reproduction of musical rhythms by children, adult musicians, and adult nonmusicians. Percept. Psychophys. 53 25- 33, 1993.

11. Fraisse, P. Time and rhythm perception. In: Handbook of Perception. VIII. Perceptual Coding, edited by E. C. Carterette and M. P. Friedman. New York: Academic, 1978.

12. Gilbert, E. N., and J . Riordan. Symmetry types of periodic sequences. III. J . Math. 5: 657-665, 1961.

13. Golomb, S. A mathematical theory of discrete classification. In: Information Theory: Fourth London Symposium, edited by C. Cherry. London: Butterworths, 1961, p 404- 425.

14. Guckenheimer, J ., and P. Holmes. Nonlinear Oscillations, Dynamical Systems and Bifurcations of Vector Fields. New York: Springer, 1983.

15. Haas, F., S. Distenfeld, and K. Axen. Effects of perceived musical rhythms on respiratory pattern. J . Appl. Physiol. 61: 1185- 1191, 1986.

16. Handel, $\mathbf{S}$. The differentiation of rhythmic structure. Percept. Psychophys. 52: 497- 507, 1992.

17. Hildebrandt, G. The autonomous time structure and its reactive modifications in the human organism. In: Temporal Disorder in Human Oscillatory Systems, edited by L. Rensing, U. an der Heiden, and M. C. Mackey. Berlin: Springer, 1987, p. 160- 175.

18. J ones, M. R. The patterning of timeand its effects on perceiving. Ann. NY Acad. Sci. 423: 158-167, 1984.

19. Kenner, T., H. Pessenhofer, and G. Schwaberger. Method for analysis of the entrainment between heart rate and ventilation rate. Pflügers Arch. 363: 263-265, 1976.

20. Kubik, G. Zum Verstehen afrikanischer Musik. Aufsätze. Leipzig, Germany: Reclam, 1988.

21. Kubik, G. Theory of African Music. Wilhelmshaven, Germany: Noetzel, 1994, vol. I.

22. Kurths, J ., A. Voss, P. Saparin, A. Witt, H. J . Kleiner, and N. Wessel. Quantitative analysis of heart rate variability. Chaos 5 88-94, 1995.

23. Longuet-Higgins, H. C., and C. S. Lee. The perception of musical rhythms. Perception 11: 115-128, 1982.

24. Nketia, J . H. K. TheMusic of Africa. New York: Norton, 1974.

25. Petsche, H., A. von Stein, and O. Filz. EEG aspects of mentally playing an instrument. Brain Res. Cogn. Brain Res. 3: 115- 123, 1996.

26. Pincus, S., and B. H. Singer. Randomness and degrees of irregularity. Proc. Natl. Acad. Sci. USA 93: 2083-2088, 1996.

27. Povel, D. J . Internal representation of simple temporal patterns. J . Exp. Psychol. Hum. Percept. Perform. 7: 3- 18, 1981.

28. Povel, D. J ., and P. Essens. Perception of temporal patterns. Music Percept. 2: 411-440, 1985.

29. Raab, E., and H. E bner. Rhythm and musical perception. The affective impression of unanimous rhythmic structures of different complexities. Z. Exp. Angew. Psychol . 29: 315- 342, 1982. 
30. Raschke, F. Automatic pattern recognition of the onset of respiratory phases using thermistor techniques. In: Proceedings of the Fourth International Symposium on Ambulatory Monitoring and the Second Gent Workshop on Blood Pressure Variability, edited by F. D. Stott, E. B. Raftery, D. L. Clement, and S. L. Wright. London: Academic, 1982, p. 345- 358.

31. Safranek, M., G. Koshland, and G. Raymond. Effect of auditory rhythm on muscle activity. Phys. Ther. 62: 161-168, 1982.

32. Schäfer, C., M. G. Rosenblum, J . Kurths, and H. H. Abel. Heartbeat synchronized with ventilation. Nature 392: 239-240, 1998.

33. Schreiber, T., and A. Schmitz. I mproved surrogate data for nonlinearity tests. Phys. Rev. Lett. 77: 635-638, 1996.

34. Schulze, H. H. Categorical perception of rhythmic patterns. Second Workshop on Rhythm Perception and Production, Marburg, September 29-October 2, 1987. Psychol. Res. 51: 10-15, 1989.

35. Senghor, L. S. L'esprit de la civilisation ou les lois de la culture Négro-Africaine. PrésenceAfricaine8/10: 51-64, 1956.
36. Summers, J . J ., R. Bell, and B. D. Burns. Perceptual and motor factors in the imitation of simple temporal patterns. Second Workshop on Rhythm Perception and Production. Marburg, September 29-October 2, 1987. Psychol. Res. 51: 23-27, 1989.

37. Van der Molen, M. W., R. J . Somsen, and J . R. J ennings. Does the heart know what the ears hear? A heart rate analysis of auditory sel ective attention. Psychophysi ol ogy 33: 547-554, 1996.

38. Van Leeuwen, P., H. Bettermann, U. an der Heiden, and H. C. Kümmell. Circadian aspects of apparent correlation dimension in human heart rate dynamics. Am. J . Physiol. 269 (Heart Circ. Physiol. 38): H 130-H134, 1995.

39. Voss, A., K. Hnatkova, N. Wessel, J . Kurths, A. Sander, A. Schirdewan, A. J . Camm, and M. Malik. Multiparametric analysis of heart rate variability used for risk stratification among survivors of acute myocardial infarction. Pacing Clin. Electrophysiol. 21: 186- 192, 1998.

40. Zatorre, R. J ., A. R. Halpern, D. W. Perry, E. Meyer, and A. C. Evans. Hearing in the mind's ear: a PET investigation of musical imagery and perception. J . Cogn. Neurosci. 8: 29-46, 1996.

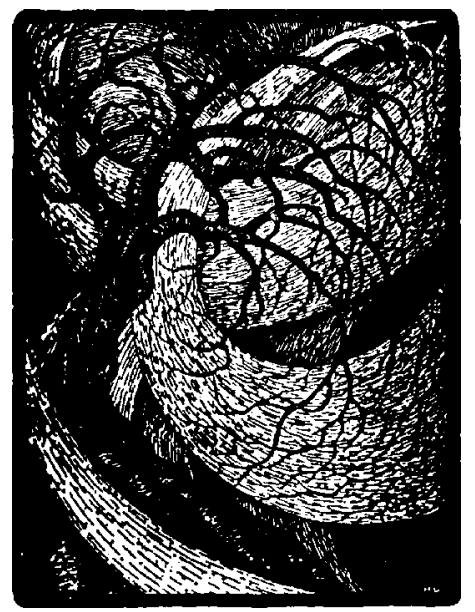

